\title{
Assembly and Phase Transitions within Colloidal Crystals
}

\author{
Bo $\mathrm{Li}^{1}{ }^{1}$ Di Zhou, ${ }^{1,2}$ and Yilong Han ${ }^{1, *}$ \\ 1, Department of Physics, Hong Kong University of Science and Technology, Clear Water Bay, Hong Kong, China. \\ 2, Multi-disciplinary Materials Research Center, Frontier Institute of Science and Technology, \\ Xi'an Jiaotong University, Xi'an 710049, China.
}

(Dated: November 10, 2017)

\begin{abstract}
Micrometre-sized colloidal particles can be viewed as large atoms with tailorable size, shape and interactions. These building blocks can assemble into extremely rich structures and phases, in which the thermal motions of particles can be directly imaged and tracked using optical microscopy. Hence, colloidal particles are excellent model systems for studying phase transitions, especially for poorly understood kinetic and non-equilibrium microscale processes. Advances in colloid fabrication, assembly and computer simulations have opened up numerous possibilities for such research. In this Review, we describe recent progress in the study of colloidal crystals composed of tunable isotropic spheres, anisotropic particles and active particles. We focus on advances in crystallization, melting and solidsolid transitions, and highlight challenges and future perspectives in phase-transition studies within colloidal crystals.
\end{abstract}

Micrometre-sized colloidal particles can assemble into numerous structures and phases (FIG.1), and are excellent model systems for the study of phase transitions [1 6 because their thermal motions can be directly visualized by optical video microscopy [7] and tracked by image processing [8. By contrast, the small spatial and timescales of atomic motions make the microscopic kinetics during phase transitions difficult to resolve.

Phase transitions are ubiquitous in nature and industry, and have an important role in materials science, statistical physics, cosmology, biophysics, chemistry and earth science. They depend strongly on dimensionality, surface properties, defects, heating and cooling rates, and external fields. Phase transitions can be classified into first-, secondand infinite-order (in which the free energy is an infinitely differentiable exponential function such as the KosterlitzThouless (KT) transition). The equilibrium behaviour of continuous phase transitions, including second- and infinite-order transitions, can be well described theoretically; however, the fundamental theory to describe first-order phase transitions is lacking. The kinetics of first-order and continuous phase transitions are difficult to predict [9].

Colloidal particles can be viewed as large atoms with tailorable size, shape and interactions [6]. Although colloids and atoms differ in some aspects (BOX 1), their phase transitions share many similarities; for example, they both follow classical nucleation theory (CNT) at weak supersaturations 10 and deviate from CNT at strong supersaturations 11. Hence, colloids can provide general information about phase transitions [4 6 . Besides being important as micrometre-scale analogues of atoms, colloids are interesting in their own right. In the past two decades, colloidal model systems have provided vital insights into the microscopic kinetics of melting, crystallization, glass transitions and solid solid transitions. Recent breakthroughs in colloidal fabrication, assembly and computer simulations open new opportunities for phase-transition studies.

Comparison between colloidal particles and atoms:

Both colloidal particles and atoms experience strong thermal motions, which enable them to form thermally equilibrated phases. The phase behaviour of colloids with weak interactions, such as hard spheres, is mainly determined by the volume fraction, $\phi$, which is analogous to the effective inverse temperature, $1 / T$, in atomic systems. Both a colloidal particle and an atom have $\sim k_{B} T$ thermal energy according to the equipartition theorem, but their diameters are very different (by a factor of $10^{3}$ or more). Consequently, the energy density and elastic moduli of 3D colloidal crystals are $10^{9}$ times smaller than those of atomic crystals (that is, they are 'soft' materials). Unlike atoms, which exhibit ballistic motion in vacuum, colloidal particles undergo overdamped Brownian diffusion without a well-defined velocity. Colloidal particles dispersed in liquid have complex long-range hydrodynamic interactions in 3D, which influence the kinetics but not the equilibrium behaviour 12 15. Note that a liquid solution is needed; otherwise microparticles form powders without Brownian motion. Quantum effects are usually negligible in colloids. Finally, colloidal particles can be made active with self-propelled motions, whereas atoms are passive.

In the first part of the Review, we describe conventional colloidal crystals composed of isotropic spheres that mimic simple atomic crystals, non-conventional colloidal crystals composed of anisotropic particles that resemble atoms with directional interactions or molecules, and crystals of active particles that have no counterpart in atomic systems. In the second part of the Review, we discuss recent studies of crystallization, melting, solidsolid transitions and other phase transitions in crystals composed of isotropic particles. Transitions within crystals composed of anisotropic or active particles are also briefly discussed. Owing to the limited space, we have highlighted only a limited number of 

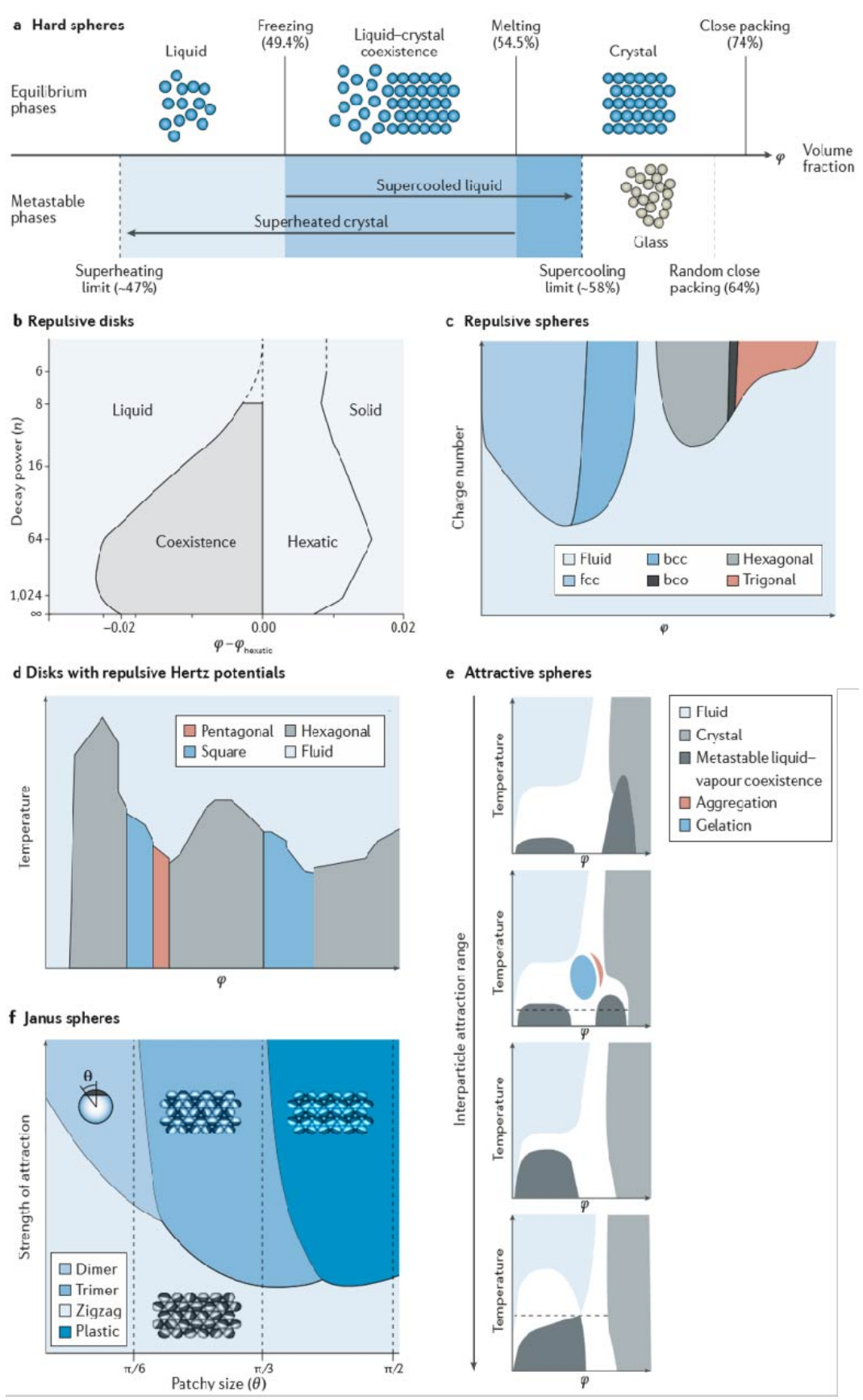

FIG. 1: Phase diagrams of monodispersed colloids. (a) Hard spheres. (b) Repulsive disks with pair potential $U(r) \sim r^{-n}$. The liquidhexatic coexistence is a signature of first-order phase transitions. (c) Spheres with long-range repulsions for ionic colloidal crystals. (d) Spheres with repulsive Hertz potentials show an infinite number of cycles of 2D crystal phases. (e) Spheres with different attraction ranges. (f) Janus spheres with different patch sizes $\theta$ and interaction strengths. $\phi$, volume fraction; $\phi_{\text {hexatic }}$, volume fraction at the onset of the hexatic phase; bcc, body-centred cubic; bco, body-centred orthorhombic; fcc, facecentred cubic. Panel b adapted with permission from REF.154, American Physical Society. Panel c adapted with permission from REF.19, American Physical Society. Panel d adapted with permission from REF.21, Royal Society of Chemistry. Panel e adapted with permission from REF.5, American Association for the Advancement of Science. Panel f adapted with permission from REF.92, Royal Society of Chemistry.

examples of this rapidly expanding research field and focus on micrometre-sized particles. 


\section{COLLOIDAL CRYSTALS}

\section{A. Crystals composed of isotropic spheres}

Different particle shapes and interactions give rise to different packing entropy, $S$, and interaction energy, $U$, in minimizing the free energy, $F=U-T S$, where $T$ is temperature, thus resulting in different phases at equilibrium.

As the simplest colloid, monodisperse (that is, uniform size and properties) hard spheres can pack into face-centred cubic (fcc), hexagonal close packed (hcp) and random hexagonal close packed (rhcp) crystals and glasses in 3D [16 18]. Face-centred cubic packing makes most efficient use of the 3D space, and thus spheres have more effective room to move; that is, they have greater free-volume entropy, which can compensate for the extra configurational entropy of random packing. Consequently, the fcc crystal is the equilibrium phase with the maximum $S$ (that is, minimum $F$ [19]) because $U=0$ for hard spheres (FIG. 1a). In practice, however, hard spheres can be trapped in a supercooled liquid or a glassy state because the 3D locally favoured packing is a tetrahedron of four spheres that cannot tile the 3D space. In general, such frustration between locally and globally favoured packing can dynamically arrest the system into a disordered structure [4. By contrast, hard or soft repulsive disks can form crystal and hexatic phases (FIG. 1b), but can not form a glassy phase in $2 \mathrm{D}$ even under fast quenching because the local optimal packing is an equilateral triangle of three disks which, can fully tile the 2D space. Crystals in lower dimensions have more long-wavelength fluctuations [20, fewer neighbours to bind each particle on its lattice site and fewer interparticle 'springs' connected in parallel rather than in series. Hence, crystals are too soft to exist in 1D unless there are long-range interactions; 2D is the critical dimensionality in which crystals are very soft with only quasi-long-range translational order and long-range orientational order. Between 2D crystals and liquids, there may exist a hexatic phase characterized by short-range translational order and quasi-long-range orientational order 20. Hard spheres under quasi-2D confinement can form triangular, square and buckled thin-film crystals [21, 22].

Soft particles that is, particles with long-range repulsions can form fcc, body-centred cubic (bcc) or other lattices 23. 24 (FIG. 1c). Interestingly, continuously compressing soft Hertz-potential spheres can induce not only quasicrystals with five-fold symmetry but also infinite cycles of hexagonal and square crystals in 2D 25] (FIG. 1d), and infinite cycles of fcc, bcc, hexagonal, simple cubic and body-centred tetragonal (bct) crystals in 3D [26. Pressure can further deform particle shape and induce new forms of crystals [27]. Experimentally, particles can be softened by grafting long polymers, reducing the crosslinker density in microgels [28] or reducing the ionic strength to increase the Debye length of the screened Coulomb interaction for charged particles 223, 24, 29. Complex repulsions involving two or more length scales can result in various superlattices [30] and quasicrystals [31 33].

Attractive colloidal particles can better mimic atoms. Attractive van der Waals forces universally exist among colloidal particles. The van der Waals force is strong over short distances $(<100 \mathrm{~nm})$ for microparticles and induces aggregation. To avoid such coagulation, van der Waals attractions are usually weakened below kBT by grafting polymers or surfactants to form a low-density surface (steric stabilization) or screened by electrostatic repulsions for charged particles (charge stabilization) [34. Beyond the van der Waals attraction range, typical interparticle attractions include depletion attraction induced by adding small particles [35-38, and Casimir-like or wetting attraction induced by the fluctuation of either the two-component liquid near its critical point or percolation clusters of small particles 39 42. Both the strength and range of attraction affect the phase behaviour (FIG. 1e). For example, when the range of attraction is much smaller than the repulsive core, particles form a fluid without distinguishable liquid and gas phases in colloida [5] and molecular systems such as buckyballs [43.

Unlike repulsive or long-range attractive colloidal spheres, which can easily form large crystals, shortrange attractive colloids often form disordered aggregates or gels 4] (FIG. 1e), indicating a rugged free-energy landscape. This can be explained in terms of the competition between locally and globally favoured structures [44. When a liquid-like particle crystallizes, its neighbours must move to make way for new neighbours. Neighbours moving radially under short-range attraction require high energy, and tangential movement is often forbidden because of the constraints from neighbours' neighbours. Hence, the system tends to be deeply trapped in a disordered state.

Unlike atoms, colloidal particles cannot be perfectly identical in size or other properties. This polydispersity is a generic feature of colloids that can hinder crystallization and even induce new types of crystals [45 48. Hard spheres with $6 \%$ polydispersity can fractionate into crystallites with different mean diameters and undergo a re-entrant melting transition [45. Hertz spheres with a polydispersity $<6 \%$ can form normal crystals at high volume fractions, $\phi$, and distinct disordered crystals at low $\phi$ [46]. Uniformsized spheres with different softnesses can pack into another type of disordered crystal with perfect lattices and glassy behaviour [47, 48.

Compared with nanoparticles, microparticles do not self-assemble into high-quality crystals as easily, and defects are common. Hence, it is necessary to direct assembly by using an external force [49 52] (FIG. 2ad) or different liquid solvents (FIG. 2eg), or, in the case of more complex crystals, by using bidisperse spheres [29, 53] (FIG. 2hj), competing interactions [30. (for example, short-range attraction versus long-range repulsion) (FIG. 2k), templating [54] or a combination thereof. After assembly, defects can be effectively annealed away by cycling tunable colloidal 

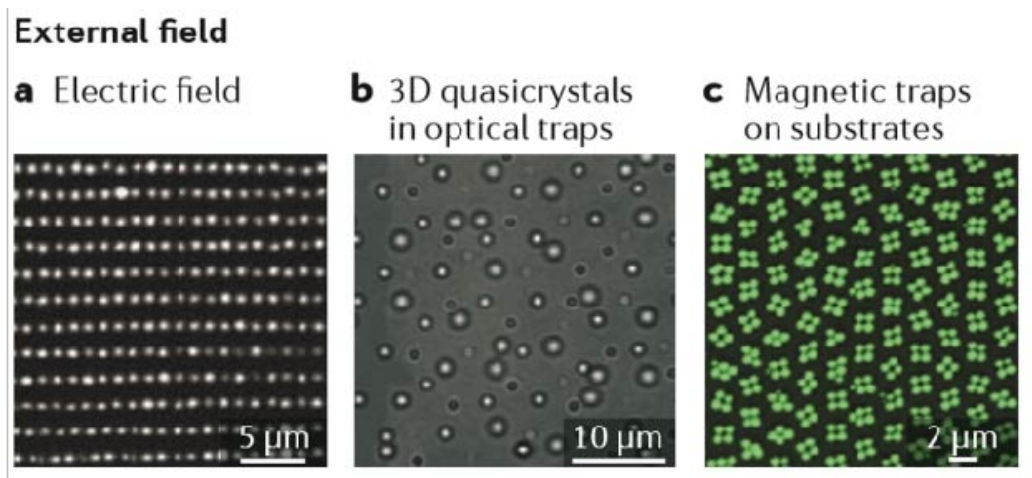
d Temperature gradient

\section{Liquid solution}

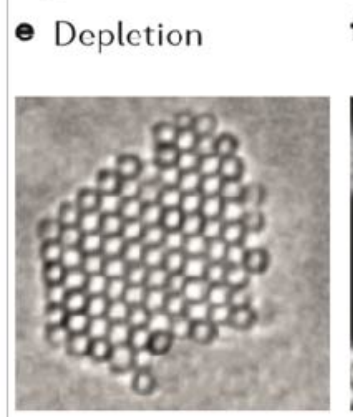

f Capillary attraction

g Casimir-like attraction on a curved surface
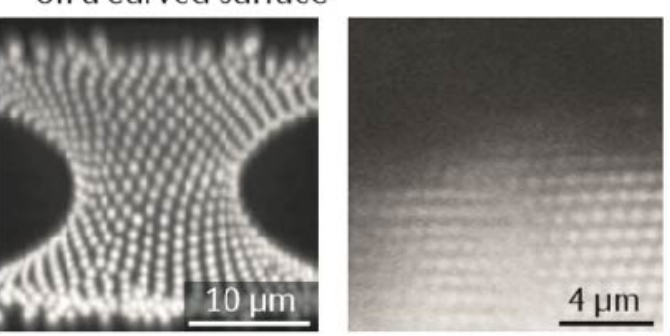

\section{Bidisperse colloids}

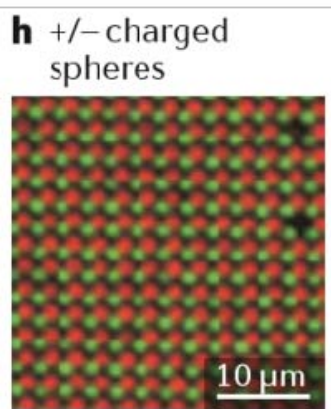

i Big/small spheres

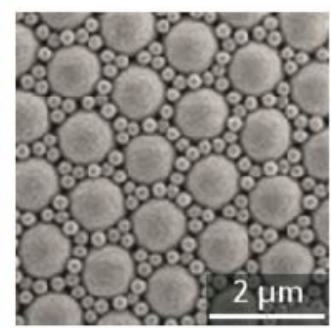

j DNA-coated spheres

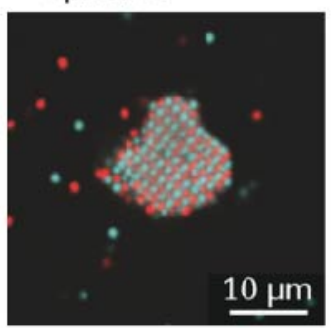

\section{Competing interactions}

k Capillary, dipolar and hydrophobic interactions

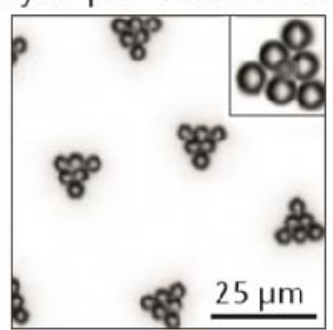

FIG. 2: Strategies used to assemble isotropic particles into crystals. (a-d) Application of external fields. (e-g) Adjustment of the liquid solution. (h-j) Use of bidisperse spheres. (k) Competing interactions. Image a adapted with permission from REF.43, American Physical Society. Image b adapted with permission from REF.45, Optical Society of America. Image c from REF.50, Nature Publishing Group. Image d adapted with permission from REF.46, Optical Society of America. Image e from REF.32, Proceedings of the National Academy of Sciences. Image f from REF.143, Nature Publishing Group. Image g from REF.34, Nature Publishing Group. Image h from REF.25, Nature Publishing Group. Image i adapted with permission from REF.47, WileyVCH. Image $\mathrm{j}$ adapted with permission from REF.95, American Association for the Advancement of Science. Image $\mathrm{k}$ adapted with permission from REF.26, American Physical Society.

crystals near their melting points [10, 55], by shaking crystals in external fields [55] 57] or with holographic optical tweezers [58].

\section{B. Crystals composed of anisotropic particles}

Much of the interest in colloidal assembly comes from its potential applications for photonic crystals [59], and recent progress made in fabricating crystals composed of anisotropic particles is particularly valuable in this respect. Particle synthesis techniques have been used for the past three decades to produce micrometre-sized, non-spherical colloidal particles, such as rods, platelets, convex and concave polyhedrons, and spheres with bulges or dents [6063]; however, the phase-transition behaviour of such particles has rarely been studied. Recently, progress has been 
made in producing active particles [64, 65, anisotropic particles [63, 65, 66] and particles with tunable interactions 34 36, 39, 67, 68. These experimental breakthroughs have greatly expanded our knowledge of anisotropic-particle assembly, and led to the computational prediction of diverse structures 69 71], although many of these have yet to be realized experimentally. Recent reviews of colloid fabrication are available in the literature [65, 66, in this Review we focus on crystal assembly [71 74].

Hard ellipsoids are a simple type of anisotropic particle, which can be stretched from spheres and can assemble into rotator crystals (that is, plastic crystals), liquid-crystal phases and crystals in 2D [75] and 3D [76] however, experimental realization remains challenging because micrometre-sized ellipsoids tend to be trapped in glassy states 77. By contrast, using an electric field, long-range repulsive rods can be tuned from a liquid to a rotator crystal [78. (FIG. 3a) to a crystal without vitrification because of their abundant free space. Photolithography can produce numerous platelets with arbitrarily designed 2D shapes [79 82 , which suffices for assembly in 2D but not in 3D. Monodispersed squares, triangles, pentagons and square crosses (FIG. 3b) were assembled into various 2D crystals by slightly tilting samples under gravity [80 82. For example, square platelets form a hexagonal rotator phase and a rhombic crystal as density increases, but do not form any phase with four-fold symmetry [80. Non-chiral triangles can form a crystal with left-handed and right-handed chiralities either mixed locally in experiments 80 or separated into two large domains in simulations [83. These platelets do not have tunable sizes or interactions; hence, it remains a challenge to drive a phase transition and study its kinetics.

Polyhedra [84, 85] have been fabricated and assembled into crystals (FIG. 3c) [86]. The experimental study of colloidal crystals is more challenging in $3 \mathrm{D}$ than in $2 \mathrm{D}$ because it requires many more particles and because refractiveindex matching between particles and the liquid solution is required for 3D confocal imaging. Hence, simulations take the leading role in studies of 3D assembly of anisotropic particles [71, 72. Numerous types of crystals, plastic crystals, liquid crystals and amorphous phases were revealed in simulations for 145 types of hard polyhedrons by systematically varying their shapes 69 . This demonstrates that a purely entropic effect can give rise to almost any structure observed in atomic systems, even for simple hard polyhedrons.

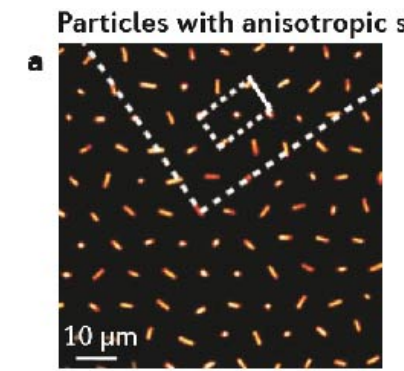

Active colloids

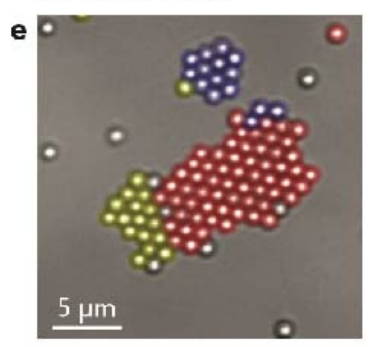

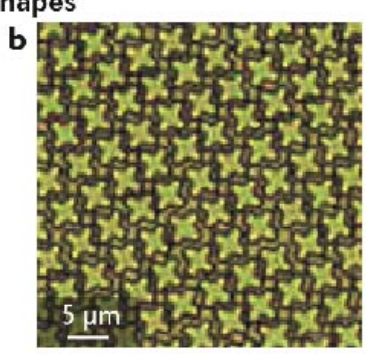

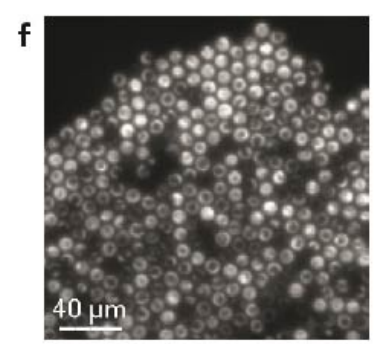

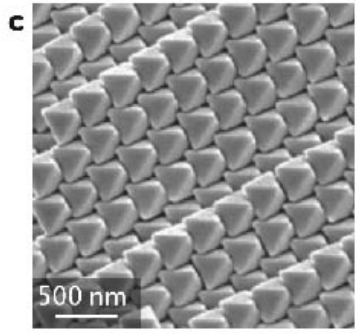
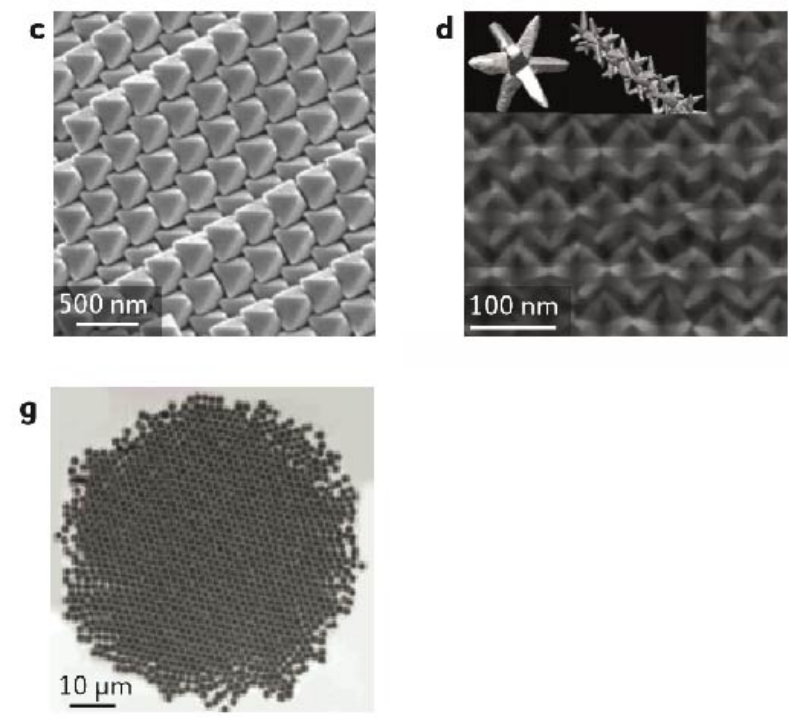

FIG. 3: Colloidal crystals composed of anisotropic or active particles. (a) Rods with long-range repulsions form a rotator crystal with random orientations. (b) A 2D crystal assembled from square crosses. (c) Octahedra packing in 3D. (d) Hierarchical assembly of nano-octapods. (e) Self-propelled Janus spheres self-assemble into crystallites. (f) Spherical selfrotating Thiovulum majus bacteria self-assemble into a crystal with global random rotation. (g) Paramagnetic Janus spheres spin under a rotating magnetic field and form a crystallite. Panel a from REF.74, Nature Publishing Group. Panel b adapted with permission from REF.77, Institute of Physics. All rights reserved. Panel c from REF.185, Nature Publishing Group. Panel d from REF.82, Nature Publishing Group. Panel e adapted with permission from REF.111, American Association for the Advancement of Science. Panel f adapted with permission from REF.109, American Physical Society. Panel g reproduced with permission from REF.110, Royal Society of Chemistry.

Compared with convex particles, concave particles can form more complex and even hierarchical structures [87. For example, octapod-shaped nanoparticles interlock to form chains, which in turn stack to form a 3D super lattice with high porosity and rigidity [87] (FIG. 3d). Such hierarchical assembly greatly expands the possible structures.

Besides shape anisotropy, interaction anisotropy can give rise to richer crystalline structures [71, 72, 74, which 
in recent years have been made accessible by breakthroughs in the fabrication of patchy spheres [88 and DNAcoated particles [68. A simple type of particle with anisotropic interactions is the Janus sphere, which comprises two hemispheres with different properties, for example, half latex and half metal 89. In the past three decades, various coating methods for making Janus [89] and multipatch spheres [90 have been developed. Patch-induced anisotropic interactions and different responses to external fields give rise to complex behaviour; however, novel lattices have only been experimentally achieved using triblock spheres with attractive hydrophobic patches. Surprisingly, these spheres formed a kagome lattice 91] rather than a typical hexagonal lattice because of the rotational entropy of the spheres [92]. Simulations of various patchy particles have led to the discovery of new crystals [93 95] and new phase diagrams 94 96. (FIG. 1f), and the extraction of some general features; for example, directional interactions can promote hierarchical assembly [97, 98.

Base-pair sequences in DNA can be programmed in biotechnology, allowing single-strand DNA to be used as glue with programmable base-pair binding sites. Although programmable anisotropic interactions are promising, micrometre-sized spheres have only been uniformly coated with DNA and thus have isotropic attractions. Colloidal spheres coated with complementary DNA sequences have temperature-sensitive attractions and can form crystallites 99, 100, (FIG. 2j). DNA-coated nanospheres can form large 3D crystals 101, but DNA-coated microspheres usually form aggregates or small crystallites [99, 100 because their attraction ranges relative to the repulsion cores are extremely short 99,100 . In addition, directional binding lowers both energy and entropy, resulting in an abnormal free-energy minimum corresponding to small crystallites; further crystallite growth is prohibited by the higher freeenergy barrier [102]. Hence, the global free-energy minimum of large crystals is kinetically inaccessible. Quantitative analysis [102, 103] has led to the development of important protocols to fabricate large crystals, and experimental techniques are reaching maturity after two decades of [178. Consequently, DNA-coated spheres now have great potential to be assembled into new, designer structures (see REF. 178] for a recent review on this subject).

Modern simulation techniques can be used for efficiently exploring the large parameter space of anisotropic building blocks and their packing structures [69, 71, 72, 96, 104, and, in turn, could reveal general rules governing their assembly and structure. In the past decade, simulations have played an important part in, and provided an indispensable understanding of, anisotropic particle assembly [71, 72].

Besides colloids with anisotropic interactions, topological colloids in liquid crystals, such as rings [105] or spheres with topological interacting knots [106, represent a new class of interaction and assembly, and could form new crystalline structures [106.

\section{Crystals composed of active particles.}

'Active matter' contains self-driven units by converting stored or ambient energy into systematic movement, and includes animal groups, energy-consuming polymers and active colloids 64]. Active particles are different from atoms: they can exhibit new phenomena and new states of matter. Active matter is a rapidly expanding area as a new platform to study non-equilibrium physics. In the past decade, colloids have been made active with translational or rotational self-propulsion by adding local chemical, temperature, electric or force gradients around individual particles. Examples include chemical-reaction-driven Janus particles, thermophoretic Janus particles, and the use of force gradients induced by surface tension or polymerization [74, 107. Self-propelled spheres can spontaneously form 2D triangular lattices (FIG. 3e) because a newly crystallized particle is buried by subsequently deposited particles before its rotation can align it in the direction needed to escape from the crystal [108. This self-trapping mechanism is distinct from the crystallization of passive particles. In addition, self-rotating bacteria 109] (FIG. 3f) and Janus paramagnetic spheres under a rotating magnetic field [110] (FIG. 3g) can assemble into a rotating crystal via hydrodynamic interactions. Current active-particle studies mainly focus on low-density 2D gases and small crystallites [11], whereas dense [112] and $3 \mathrm{D}$ active systems are desirable for phase-transition studies in the future.

\section{PHASE TRANSITIONS}

The kinetics of a phase transition can affect the transition rate, the intermediate state and even the final structure; however, kinetic pathways are difficult to predict, owing to the complex high-dimensional free-energy landscape. Measurable kinetic pathways only exist near equilibrium, where a small number of collective coordinates are sufficient to describe the kinetic process. Quasi-equilibrium can be expected to hold if bond breaking and formation are much shorter than the nucleation timescale. First-order phase transitions typically follow the nucleation mechanism, which can be roughly divided into three stages: incubation, critical nucleus formation and growth of the post-critical nucleus. The small scales of the first two stages require measurement using optical microscopy (representative images of the different transitions are shown in FIGS. 4,5), whereas the third stage was often studied by light scattering in earlier 
colloid experiments [1, 113]. First-order transitions can be identified, for example, from the coexistence of two phases at equilibrium, a van der Waals loop in the free energy, hysteresis, an order-parameter jump or different asymptotic behaviours of susceptibility [9. Strictly speaking, phase transitions are defined at the thermodynamic limit. Most colloidal crystals in phase-transition studies contain millions of particles, thousands of which are in the field of view. Transitions within small colloidal crystallites can cast light on nanocrystal transformations, which are interesting in their own right.

\section{Gibbs free energy of nucleation:}

In classical nucleation theory, the Gibbs free energy of nucleation is expressed as:

$$
\Delta G=-V \rho \Delta \mu+A \gamma+E_{\text {strain }}-E_{\text {defect }},
$$

where $V$ : volume

$A$ : area

$\gamma$ : surface tension

$\rho$ : number density of particles in the nucleus

$\Delta \mu(>0)$ : chemical potential difference between the parent phase and the nucleus

$E_{\text {strain }}$ : strain energy caused by volume change of the nucleus; zero for fluid parent phase and finite for solid parent phase

$E_{\text {defect }}(>0)$ : energy of preexisting defects in volume $V$ of a crystalline parent phase

CNT is most widely used to interpret experimental and simulation data 114. In CNT (BOX 2), competing terms with opposite signs give rise to a barrier in $\Delta G$ (the Gibbs free energy of nucleation) that is a function of the nucleus size. Thus, small nuclei tend to shrink rather than grow, unless their size exceeds a critical value related to the barrier height of $\Delta G$ (red curve in FIG. 5a). The energy of pre-existing defects or surfaces ( $\left.E_{d} e f e c t\right)$ reduces $\Delta G$. Thus, nucleation tends to start heterogeneously from defects or surfaces.

CNT is based on certain assumptions. For example, it is assumed that the nucleus surface is rough and that growth takes place without the existence of preferential sites; that nuclei grow through monomer attachment rather than collective attachment of multiple particles; and that growth occurs about a single nucleus without coalescence of nuclei or precursors. These assumptions usually hold at weak super saturation; however, the deviations that occur at strong supersaturation11 are less clear. One research focus in nucleation is non-CNT behaviour, such as in the following three cases. First, the parent phase develops certain structures or dynamics before nuclei form. Second, the nucleus can evolve through intermediate metastable states with lower free-energy barriers rather than by directly transforming into the product phase [97, 115] (blue curve in FIG. 5a), which is called Ostwald's step rule. This rule, in principle, can be generally applied to any barrier-crossing process in a rugged landscape, such as protein folding and chemical reactions. In general, the intermediate metastable state is more likely to appear if particles have appropriate interaction ranges or possess different types of interactions that stabilize the different phases [97. Third, instead of $\Delta G(n)$ for a nucleus with $\mathrm{n}$ particles in CNT, the nucleation may have additional collective coordinates, $\mathrm{m}$ (for example, crystallinity, number of particles in the intermediate phase [116], space curvature [117]), which fall within multidimensional reaction-rate theory. The kinetics follows the steepest pathway through the saddle point of the free-energy surface $\Delta G(n, m)$ (FIG. 5b). Multiple paths can occur simultaneously 118 if their barrier heights are similar. More collective coordinates often yield a high-dimensional rugged landscape with many basins, similar to those shown in FIG. 5c. Note that basins may not be fully surrounded by barriers in higher dimensions; that is, they have narrow barrier-free channels connected to lower-energy states. Such morphology should be more prevalent for larger systems that have extremely high-dimensional landscapes. In addition to CNT, alternative theories can describe nucleation and provide different predictions [119, 120.

Tunable colloids enable us to drive a phase transition quasi-statically and cycle through the transition multiple times for better statistics. Temperature on a microscope stage usually cannot be tuned far from $300 \mathrm{~K}$ to change the thermal energy $k_{B} T$; hence, colloids are usually tuned by changing the particle interaction. Fabricating tunable colloids is one of the key breakthroughs of the past decade: examples are repulsive poly(N-isopropylacrylamide) (pNIPAM, NIPAM or NIPA) spheres with temperature-sensitive diameters [10, 11, 55, 67, 121]; paramagnetic spheres [56] or non-magnetic spheres in ferrofluids, for which dipolar interactions can be tuned by the magnetic field [122]; temperature-sensitive DNA-binding [99 101, 123, 124]; fluctuation-induced 39 42] attractions; and depletion [35]37] or surface absorption induced by temperature-sensitive polymers [36. NIPA shells on latex cores create ellipsoidal, faceted or bowl-shaped particles with tunable size and shape [125. Magnetic particles are ideal for assembling crystals in 2D but not in 3D, because the attractions along the magnetic field induce chains rather than crystals. Depletion-induced or fluctuationinduced attraction strength is often not finely tunable in the useful range of $0-1 k_{B} T$ for phase transitions. Adding a fraction of non-temperature-sensitive depletant can induce more finely tuned depletion attractions 37 . 
Most experiments and simulations have been focused on colloidal phase transitions in either quasi-static processes or systems abruptly changed to a supersaturated state to observe the equilibration process. These results can be compared with CNT, which describes the evolution of a supersaturated system under fixed conditions. However, phase-transition kinetics has not been well studied while changing a thermodynamic variable, such as the heating or cooling rate, and the related dynamical theories are limited for first-order [126] and second-order [127] phase transitions.

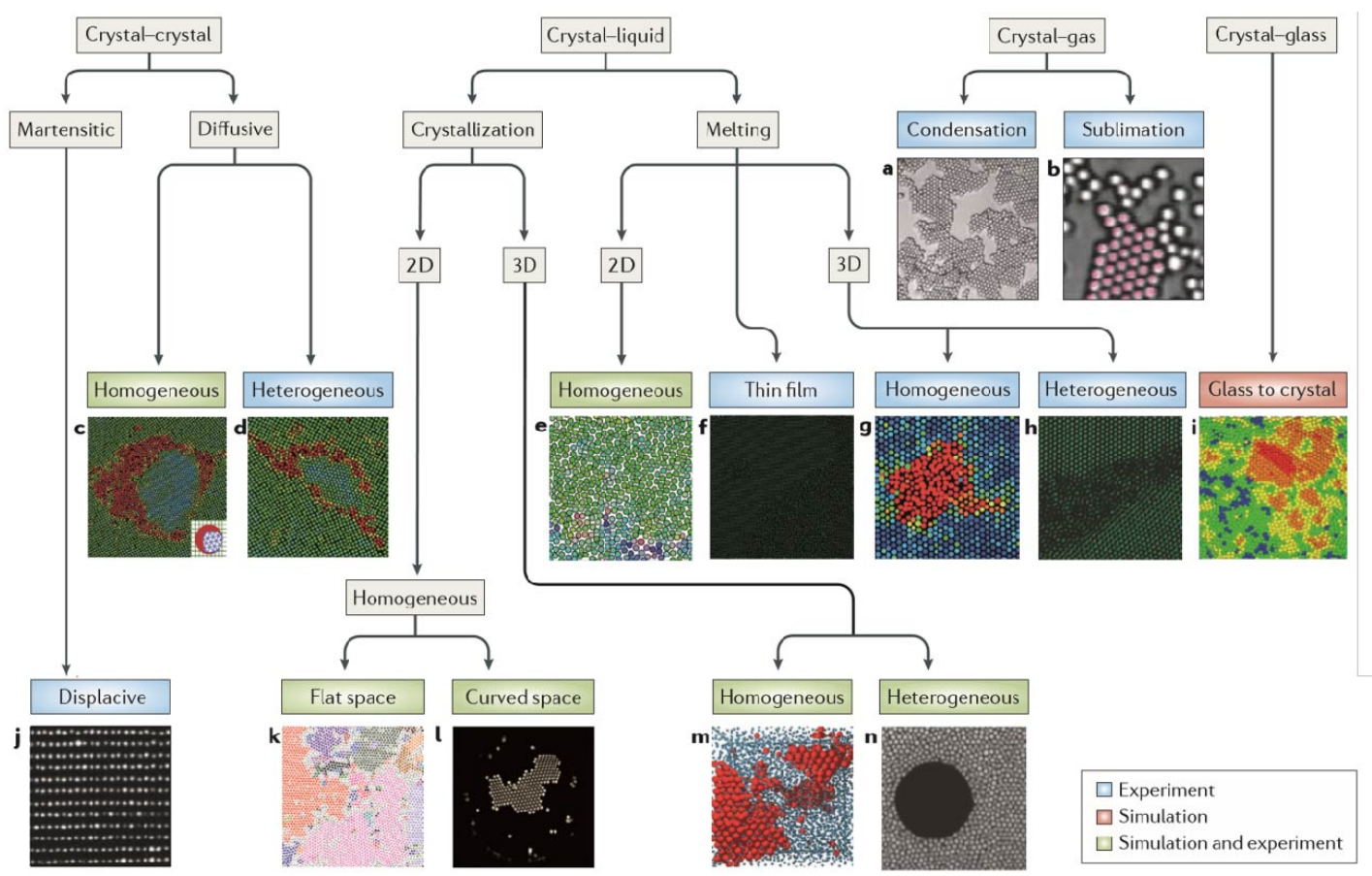

FIG. 4: Phase-transition studies within colloidal crystals in the past two decades. (a,b) Condensation and sublimation by tuning inter-particle attractions. (c,d) Transition from square to triangular lattice within a crystalline domain (panel c) and at a grain boundary (panel d). (e) 2D crystal melting. (f) Five-layer thin film melted from a dislocation and a grain boundary. (g) Melting in a defect-free region inside a 3D crystal. (h) Melting near a grain boundary inside a 3D crystal. (i) Crystallization of monodisperse hard-sphere glass. (j) Electric-field-induced fcc to bct transition. (k) Magnetic spheres crystallized to a polycrystal. (l) Crystallization on a droplet surface. (m) Crystallization inside 3D bulk supercooled liquid. (n) Crystallization from the surface of a large spherical seed. Image a from REF.64, Nature Publishing Group. Image b adapted with permission from REF.31, American Association for the Advancement of Science. Images c,d from REF.121, Nature Publishing Group. Image e adapted with permission from REF.154, American Physical Society. Image g adapted with permission from REF. 10, American Association for the Advancement of Science. Image h adapted with permission from REF.62, American Association for the Advancement of Science. Image i adapted with permission from REF.145, American Physical Society. Image j adapted with permission from REF.43, American Physical Society. Image k adapted with permission from REF.142, Proceedings of the National Academy of Science. Image l adapted with permission from REF.117, American Association for the Advancement of Science. Image m adapted with permission from REF.127, American Association for the Advancement of Science. Image n from REF.137, Nature Publishing Group.

\section{A. Crystallization}

Crystallization is the most intensively studied phase transition in colloids (see reviews on this subject [128, 129] and references therein). Homogeneous crystallization (that is, crystallization without any preferential sites) of a 3D supercooled liquid can be observed deep inside the bulk, which avoids heterogeneous crystallization at walls. In a ground-breaking confocal microscopy experiment, the first in situ observation of 3D crystallization was achieved at the single-particle level by using hard-sphere colloids, thereby allowing measurements of the nucleus structure, size distribution, nucleation rate and surface tension to be taken [130. The measured homogeneous nucleation rate was very different from the simulated values [131, which could be attributed to nucleation precursors 132 or other factors [34, 129]. Various nucleation precursors, such as the predicted bcc nucleus in transitions towards arbitrary product lattices [133], intermediate-range ordered liquids [132], dense liquid droplets [134] and ordered clusters [118], have 
been observed in different repulsive colloids. Crystallization in attractive colloids has been experimentally explored recently using DNA-coated spheres [135].

Heterogeneous crystallization in 3D has been studied on flat walls [136, 137] and patterned substrates [138, and for seed particles with different curvatures [139, 140]. The seed can be a large sphere [140] or an array of optical traps [141. Small seeds suppress crystallization, whereas large seeds promote it [139, 140. In particular, when the seed diameter is appropriate (several times that of the colloidal spheres), crystallites nucleate on the seed surface before detaching [139, 140], (FIG. 5d).

2D freezing has been much less studied than 2D melting, although it can be easily observed by reversing the tuning parameters in 2D melting experiments. Note that melting and freezing share the same equilibrium phases, but their kinetics are different and beyond the scope of 2D melting theories [142 144. The kinetic KibbleZurek mechanism in cosmology [127. successfully describes the scaling of the generation rates of topological defects, and the dynamical length scales at various quenching rates as a monolayer of paramagnetic colloidal spheres freezes [145]. This remarkable similarity between cosmology and soft-matter systems shows that certain features of the non-equilibrium kinetics of phase transitions are universal.

Phase transitions in curved spaces are of basic interest, but they are difficult to study in atomic systems. They can, however, easily be observed in colloidal particles trapped at a fluid/fluid interface [117, 146]. Unlike crystallization in flat space, colloidal spheres on a liquid droplet surface nucleate into fractal-like crystalline patches (FIG. 5e) rather than a large crystal, because of curvature-induced strain [117] (FIG. 5b).

Deeply supercooled liquids have dynamic heterogeneity, in which the viscosity can differ by orders of magnitude in neighbouring mesoscopic regions. Such inhomogeneity would certainly affect crystallization, but has rarely been considered in nucleation theories. Simulations have revealed that crystallization and dynamic heterogeneity enhance each other in 3D hard-sphere supercooled liquids and glasses [147, 148].

\section{B. Melting}

Although crystallization has been intensively studied, melting deserves separate investigation because the two processes differ in the following crucial aspects. First, melting and freezing points are usually different in colloids; for example, $\phi_{\text {melt }}=54.5 \%$ and $\phi_{\text {freeze }}=49.4 \%$ for hard-sphere crystals 16] (FIG. 1a). At $49.4 \%<\phi<54.5 \%$, crystals with $\phi=54.5 \%$ and liquids with $\phi=49.4 \%$ coexist at equilibrium. Second, tunable colloids are required when the metastable parent phase is ordered (for example, during melting [10, 67]), but not for a disordered parent phase (for example, brute force can break crystals into supercooled liquids, which can equilibrate back via crystallization without tuning [130]). Third, defects are important in melting [20, 55, 67, but are generally insignificant in crystallization. Fourth, small nuclei can have various ordered structures in crystallization [118, 128, 129, 132, 133, but only disordered liquid exists in melting. Fifth, supercooled liquids are common in nature because crystallization can be easily suppressed below the freezing temperature, but superheated crystals [10] are rare because crystals will melt from free surfaces (that is, solid/vapour interfaces) once they reach the melting point. Sixth, the melting speed monotonically increases with the degree of superheating [11, whereas the crystallization rate is non-monotonic as the temperature decreases towards the vitrification point because of the increasing driving force of crystallization and the decreasing diffusivity of the particles 129, 131. Seventh, nucleus expansion can induce strain in the parent lattice during melting, but crystallization induces no strain in the parent liquid phase. A larger liquid nucleus in melting causes more parent-lattice deformation and gives rise to a higher energy barrier. Hence, there is no kinetic pathway for melting when the driving force is weak under mild superheating [149] (black curve in FIG. 5a). This forbidden gap does not exist in crystallization. Last, after the liquid has fully crystallized, small polycrystalline grains coalesce into large ones [145. Such a ripening process in crystallization does not exist in melting.

Crystals tend to melt heterogeneously from pre-existing defects. The 'defect strength' in terms of inducing melting decreases roughly in the following order: free surface (that is, solid/vapour interface) $>$ bulk triple junction $>$ highangle grain boundary $>$ flat substrate $>$ low-angle grain boundary $>$ dislocation $>$ partial dislocation $>$ point defects (vacancy and interstitial). Stronger defects may also pre-empt melting at weaker defects. This expected trend has not, however, been well studied in colloidal systems. Heterogeneous melting at grain boundaries [67] and hard walls 150] has been experimentally observed in repulsive colloidal crystals. In nature, crystals are composed of attractive particles with free surfaces. Thus, they always melt from free surfaces because the pre-melted surface liquid serves as a huge post-critical nucleus. Surface pre-melting exists in almost all crystals [151] and has been recently observed at the single-particle level using tunable attractive colloids 152 .

Homogeneous melting only occurs in defectfree crystalline regions if melting is avoided from surfaces and defects outside the region of interest. Homogeneous 3D melting was observed at the single-particle level for the first time by focusing a beam of light to superheat the interior of a NIPA colloidal crystal while the ambient crystal remained below the melting point [10]. During the incubation stage, particles swap positions with neighbours, and this triggers 
nucleation of melting. Such a nucleation precursor, rather than defects as is commonly assumed, keeps the lattice intact [10]. Particle swapping has also been observed in 2D crystals [153. The nucleus growth kinetics agrees with CNT predictions under mild superheating, but deviates under strong superheating owing to non-CNT effects, such as nucleus shape fluctuations, nuclei coalescence and multimer attachment11. At the superheat limit where the superheated crystal crosses over from a metastable to an unstable state (blue dashed curve in FIG. 5a), the lattice becomes mechanically unstable and catastrophically breaks down[10] (FIG. 5g). This transition at the superheat limit is similar to the spinodal decomposition of crystallization at the supercool limit [154] and remains poorly understood.

The predominant theory used to describe 2D melting is the KosterlitzThoulessHalperinNelson Young (KTHNY) theory, which predicts that 2D crystals melt via two continuous KT transitions through an intermediate hexatic phase [142, 143. An alternative 2D melting scenario is the one-step first-order transition associated with the formation of grain boundaries [144. These theories do not exclude other melting scenarios. Many molecular monolayers exhibit one-step first- order melting [20], but grain boundaries have not been reported in such systems in which the substrate effects are strong. By contrast, 2D colloidal monolayers confined between two flat walls [155] or at a fluid/fluid interface [56] have negligible substrate effects, and defects can be directly observed. Melting of 2D colloidal crystals with tunable long-range 56 or short-range 155 repulsions exhibits an intermediate hexatic phase, but the order of the two transitions cannot be well resolved experimentally. Simulations reveal that the melting behaviour can be correctly resolved only for systems containing more than 106 particles in 2D [156 158. Large-scale simulations show that disks with various repulsion ranges follow the KTHNY melting scenario except that the liquidhexatic phase transition is weakly first-order for short-range repulsive disks [157, 158] (FIG. 1b).

Although 2D melting has been intensively studied in molecular and colloidal systems, the following three aspects are still poorly understood. First, the kinetics is beyond the scope of 2D melting theories [20] and has been poorly studied experimentally [145]. Second, 2D melting in attractive colloidal crystals has not been well studied [152]. Last, both KTHNY and grain-boundary- mediated melting theories are developed for infinitely large defect-free crystals without surfaces [151. Both 3D and 2D crystals with free surfaces are expected to premelt and melt from surfaces [151, although surface melting in 2D has only been indirectly measured in molecular monolayers by calorimetry [159].

Thin films are extremely valuable in technological applications, but their melting behaviour has not been thoroughly studied either theoretically or experimentally. A study of NIPA colloids confined between two walls [55, 160, showed that crystals comprising fewer than 5 layers melt catastrophically and homogeneously, 5- to 12-layer crystals melt heterogeneously from grain boundaries and dislocations (FIG. 5g), and > 12-layer crystals only melt from grain boundaries, as is the case for $3 \mathrm{D}$ crystals.

\section{Solid-solid Transitions}

Solidsolid transitions between crystals prevail in the Earth's mantle and in metallurgy. They are more diverse, complex and less understood than crystalliquid transitions. For example, water has 17 known crystalline structures but only one or two liquid phases. Solidsolid transitions are usually first-order phase transitions featuring an abrupt density change. Their kinetics typically follows either a diffusive nucleation or a martensitic transformation with concerted motion of particles. The microscopic kinetics and effect of dimensionality remain poorly understood. Most solidsolid transitions involve interparticle bond-breaking. Such reconstructive transitions are one of the least understood structural phase transitions because they are difficult to describe in theory (the difficulty lies in defining an order parameter because of the lack of a groupsubgroup relation in the lattice symmetries) [161]; in simulations, owing to sluggish dynamics; or in experiments, owing to the lack of single-particle observations in the bulk. Hence, colloids are ideal for the study of solidsolid transitions.

Solidsolid transitions have been far less studied than crystallization and melting in colloids, with most studies focused on structural properties either because the crystals were not tunable or the martensitic kinetics were too fast [4, 100, 122, 162. Solidsolid transitions in colloids have been driven by electric [49] and magnetic fields [122] and temperature-sensitive DNA attractions [100]. These systems show martensitic transformations with fast kinetics, promoted by global stretching of external fields or by small crystal size. Apart from these transitions with lattice symmetry changes, isostructural solidsolid transitions by abrupt lattice shrinking or dilation maintain the same lattice symmetry and often occur in metallic and multiferroic systems. Such transitions were discovered in simulations of attractive colloids [163] and have been experimentally confirmed [152].

Diffusive solidsolid nucleation has recently been observed in superheated thin-film NIPA colloidal crystals under local optical heating. A new type of two-step nucleation process was discovered [121]: 5-layer square lattice to liquid nucleus to 4-layer triangular lattice (FIG. 5hk). The initial nucleus is liquid because small nuclei are dominated by surface tension, which is lower at the liquid/solid interface than at the solid/solid interface. This two-step nucleation occurs in defect-free regions, near dislocations and in grain boundaries, because the constant Edefect (BOX 2) does not change the shape of $\Delta G$. This provides a clear illustration of Ostwald's step rule (blue solid curve in FIG. 5a). 


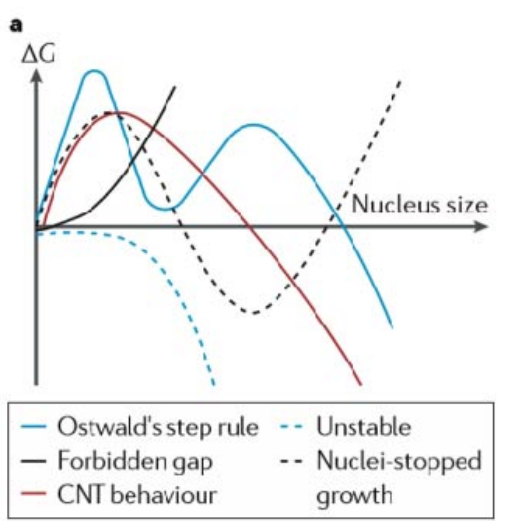

b
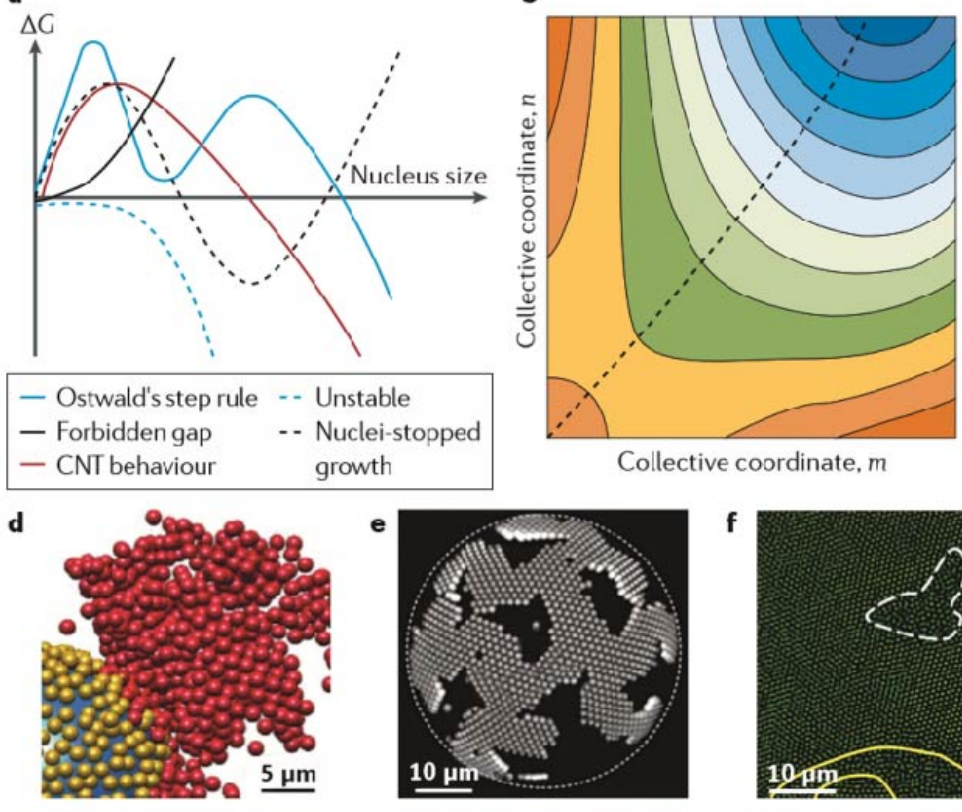

h

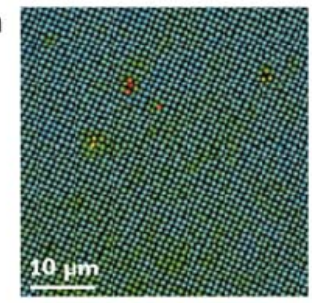

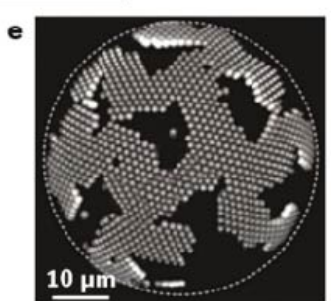

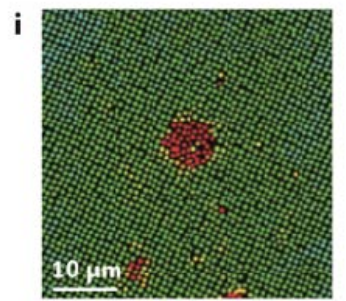

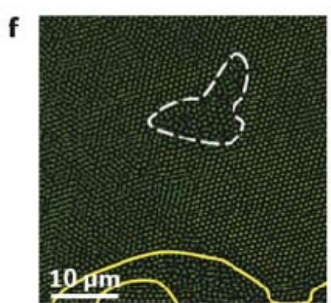

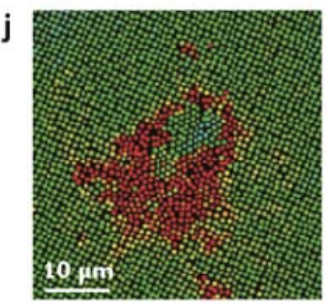

c
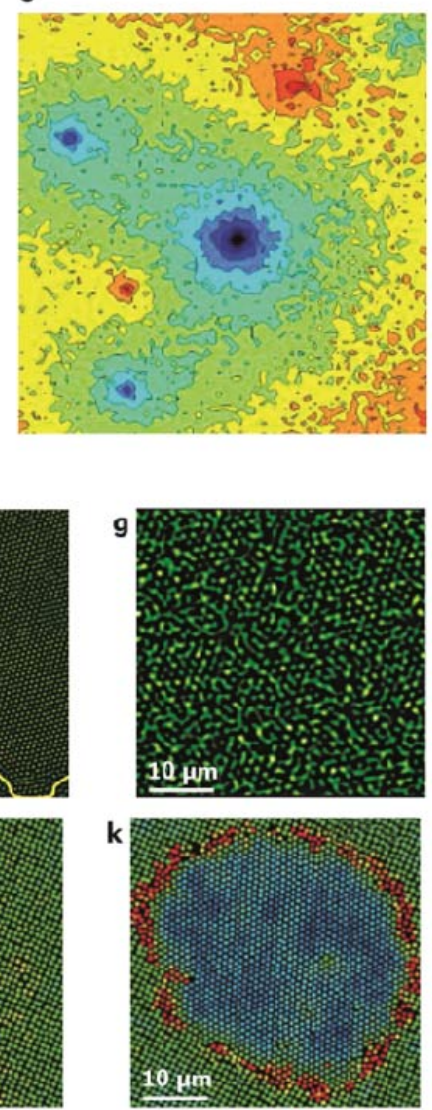

FIG. 5: Phase-transition kinetics and free-energy barriers. (a) Schematics of possible free-energy barriers. (b) Freeenergy surface as a function of two collective coordinates (such as averaged width and length of crystalline arms in panel e). (c) Short-range attractive systems often have a rugged free-energy landscape with many local minima in basins depicted by closed contours. At low temperature, the system tends to be trapped in one of the basins (producing a disordered structure) rather than reaching the global minimum (crystal). (d) A crystallite (red particles) heterogeneously nucleates from the surface of a seed (large blue sphere) and then detaches from the seed. The image is reconstructed from particle positions. (e) Homogeneous crystallization in a curved 2D space (emulsion-droplet surface) results in a fractal-like crystal. (f) Heterogeneous melting from a grain boundary and a dislocation in a five-layer thin-film crystal. (g) Catastrophic melting at the superheat limit of a 3D crystal. (h-k) The transition from a square to a triangular lattice initially develops a metastable liquid nucleus, then a triangular lattice nucleates from within the liquid nucleus. CNT, classical nucleation theory; $\Delta G$, the change in Gibbs free energy. Panel d from REF.137, Nature Publishing Group. Panel e adapted with permission from REF.117, American Association for the Advancement of Science. Panel f adapted with permission from REF.157, American Physical Society. Panel g adapted with permission from REF.10, American Association for the Advancement of Science. Panels (h-k) from REF.121, Nature Publishing Group.

The intermediate liquid has been observed in simulations of solidsolid transitions of hardsphere thin films [116] and 2D ice [164], and may exist in other atomic systems because the liquid/solid surface tension is smaller than the solid/solid surface tension for most metals and alloys. Another diffusive transition was observed when the fcc lattice of soft NIPA spheres transforms into a bct lattice driven by an electric field; however, the reverse bct to fcc transition is martensitic [165. These kinetics have not previously been proposed in atomic solidsolid systems, and thus cast new light on solidsolid transition pathways.

\section{Other phase transitions}

Other phase transitions have been significantly less studied in colloids. Crystal sublimation [35, 166] and reverse condensation [38, 40 occur only in attractive colloids. By weakening the attraction, 2D colloidal crystals sublimate from surfaces. As the size decreases to 30 particles, the whole crystallite disintegrates [35.

All the phase transitions described in the previous sections were studied in isotropic colloids. Anisotropic particles 
exhibit rich phases, but studies of their phase transitions are limited; these include the crystallization of polyhedrons [167, self-propelled particles [168], and solidsolid transitions between square and canted cubic crystallites [37. Active colloids show unique transitions [169 171] that do not exist in conventional passive thermal systems. For example, a transition from a resting to a travelling crystal in self-propelled spheres was predicted in simulations [169. In active colloids, 2D melting does not generate topological defects [170 and involves much stronger dynamic heterogeneities than do thermal systems. It was found through simulations that adding hydrodynamic interactions induces a hexatic phase, rotator crystal or glass for active rotating disks [171. Activecolloid experiments are crucial because it is challenging to take complex hydrodynamic interactions into account in simulations [171, 172].

Non-equilibrium phase transitions that feature a breakdown of detailed balance [173] are of great importance for both basic science and technology, yet are poorly understood. In colloids, non-equilibrium phase transitions have been briefly explored, including melting [174] and crystallization under fluid flow [175, 176] or temperature gradients [177. Moreover, there have been a few studies of phase transitions in active colloids [108, 168, 170]. Quantitative study of non-equilibrium phase transitions requires well-controlled conditions with a small number of collective coordinates.

\section{PERSPECTIVE}

Over the past two decades, colloidal crystals have provided remarkable insight into the microscopic kinetics of phase transitions. Studies have mainly been focused on bulk colloidal crystals composed of isotropic repulsive spheres, and on equilibrium and non-equilibrium behaviour at a fixed effective temperature. Thus, many aspects remain to be examined, for instance, the effects of surfaces, pre-existing defects and the small system size. In particular, phase transitions of surfaces and defects themselves, such as roughening transitions of free surfaces and grain boundaries, have not been explored. These aspects can be experimentally studied using currently available colloids and techniques. In metallurgy, controversies abound regarding defect dynamics, microstructure evolution and phase-transition kinetics because of the lack of single-particle resolution in the bulk. These can be studied in 'colloidal metallurgy' because metallic atoms have relatively simple interactions.

Many important processes remain to be explored in colloidal systems, including the kinetics during heating and cooling, transitions near critical points with strong fluctuations, transitions in anisotropic colloids and non-equilibrium transitions. Recent breakthroughs in fabricating anisotropic and active particles, tunable interactions and new assembly techniques provide vast possibilities for phase-transition studies. Currently, simulations take the leading role in the study of colloidal crystals composed of anisotropic or active particles, and more experimental studies are needed in the future. Researchers should try to derive general rules for how a particle's shape, interactions and activity affect crystal assembly and phase transitions. These general rules would help to achieve the goal of reverse engineering, that is, creating building blocks that will self-assemble into a prescribed structure 178, which is of fundamental importance for materials science.

Compared with the commonly used bottom-up assembly, which inevitably results in defects in crystals, more powerful top-down fabrication can directly 'print' crystals without defects or with defects in any designed pattern. Micro-platelets can be printed in ordered 2D arrays by lithography [79], but washing platelets away from the substrate disrupts the crystalline structure. Non-destructive washing would be particularly desirable in the future. In addition, current 3D printing can be performed in a liquid environment at 50- $\mu$ m resolution [179. The rapidly evolving $3 \mathrm{D}$ printing and etching technologies may give rise to a revolutionary top-down approach to fabricating colloids and colloidal crystals. New particle-tracking algorithms [180, super-resolution microscopy [181, liquid-environment transmission-electron microscopy [182, and, in particular, fast light-sheet microscopy [183, 184] could also greatly benefit phase-transition studies if implemented in colloid experiments.

This work was supported by rgc-grf16301514 and ANR/RGC-A-HKUST616/14 grants.

[1] B. J. Ackerson, Phase transitions in colloidal suspensions, CRC Press, 1990.

[2] H. Löwen, Melting, freezing and colloidal suspensions, Phys. Rep. 237, 249-324 (1994).

[3] C. A. Murray and D. G. Grier, Video microscopy of monodisperse colloidal systems, Annu. Rev. Phys. Chem. 47, 421-462 (1996).

[4] V. J. Anderson and H. N. Lekkerkerker, Insights into phase transition kinetics from colloid science, Nature 416, 811-815 (2002).

[5] D. Frenkel, Materials science, colloidal encounters: a matter of attraction., Science 314, 768 (2006).

[6] D. Frenkel, Playing tricks with designer "atoms", Science 296, 65-66 (2002).

[7] A. Kose, M. Ozaki, K. Takano, Y. Kobayashi, and S. Hachisu, Direct observation of ordered latex suspension by metallurgical microscope, J. Colloid Interface Sci. 44, 330-338 (1973). 
[8] J. C. Crocker and D. G. Grier, Methods of digital video microscopy for colloidal studies, J. Colloid Interface Sci. 179, 298-310 (1996).

[9] K. Binder, Theory of first-order phase transitions, Rep Prog Phys. 50, 783 (1987).

[10] Z. Wang, F. Wang, Y. Peng, Z. Zheng, and Y. Han, Imaging the homogeneous nucleation during the melting of superheated colloidal crystals, Science 338, 87-90 (2012).

[11] Z. Wang, F. Wang, Y. Peng, and Y. Han, Direct observation of liquid nucleus growth in homogeneous melting of colloidal crystals, Nature Communications 6, 6942 (2015).

[12] A. Furukawa and H. Tanaka, Key role of hydrodynamic interactions in colloidal gelation, Phys. Rev. Lett. 104, 245702 (2010).

[13] I. C. Jenkins, M. T. Casey, J. T. McGinley, J. C. Crocker, and T. Sinno, Hydrodynamics selects the pathway for displacive transformations in DNA-linked colloidal crystallites, Proc. Natl. Acad. Sci. U.S.A. 111, 4803-4808 (2014).

[14] M. Radu and T. Schilling, Solvent hydrodynamics speed up crystal nucleation in suspensions of hard spheres, Europhys. Lett. 105, 26001-26005 (2014).

[15] D. Roehm, S. Kesselheim, and A. Arnold, Hydrodynamic interactions slow down crystallization of soft colloids, Soft Matter 10, 5503-5509 (2014).

[16] P. Pusey and W. Van Megen, Phase behaviour of concentrated suspensions of nearly hard colloidal spheres, Nature 320, 340-342 (1986).

[17] B. Alder and T. Wainwright, Phase transition for a hard sphere system, J. Chem. Phys. 27, 1208 (1957).

[18] V. N. Manoharan, Colloidal matter: packing, geometry, and entropy, Science 349, 729-735 (2015).

[19] P. G. Bolhuis, D. Frenkel, S.-C. Mau, and D. A. Huse, Entropy difference between crystal phases, Nature 388, 235-236 (1997).

[20] K. J. Strandburg, Two-dimensional melting, Rev. Mod. Phys. 60, 161-207 (1988).

[21] A. Fortini and M. Dijkstra, Phase behaviour of hard spheres confined between parallel hard plates: manipulation of colloidal crystal structures by confinement, J. Phys. Cond. Matt. 18, L371 (2006).

[22] M. Schmidt and H. Löwen, Freezing between two and three dimensions, Phys. Rev. Lett. 76, 4552 (1996).

[23] D. Gottwald, C. Likos, G. Kahl, and H. Löwen, Phase behavior of ionic microgels, Phys. Rev. Lett. 92, 068301 (2004).

[24] A. Yethiraj and A. van Blaaderen, A colloidal model system with an interaction tunable from hard sphere to soft and dipolar, Nature 421, 513-517 (2003).

[25] W. L. Miller and A. Cacciuto, Two-dimensional packing of soft particles and the soft generalized Thomson problem, Soft Matter 7, 7552-7559 (2011).

[26] J. C. Pàmies, A. Cacciuto, and D. Frenkel, Phase diagram of Hertzian spheres, J. Chem. Phys. 131, 044514 (2009).

[27] V. M. Batista and M. A. Miller, Crystallization of deformable spherical colloids, Phys. Rev. Lett. 105, 088305 (2010).

[28] D. Heyes and A. Brańka, Interactions between microgel particles, Soft Matter 5, 2681-2685 (2009).

[29] M. E. Leunissen, C. G. Christova, A.-P. Hynninen, C. P. Royall, A. I. Campbell, A. Imhof, M. Dijkstra, R. Van Roij, and A. Van Blaaderen, Ionic colloidal crystals of oppositely charged particles, Nature 437, 235-240 (2005).

[30] A. D. Law, M. Auriol, D. Smith, T. S. Horozov, and D. M. A. Buzza, Self-assembly of two-dimensional colloidal clusters by tuning the hydrophobicity, composition, and packing geometry, Phys. Rev. Lett. 110, 138301 (2013).

[31] K. Barkan, M. Engel, and R. Lifshitz, Controlled self-assembly of periodic and aperiodic cluster crystals, Phys. Rev. Lett. 113, 098304 (2014).

[32] M. Engel, P. F. Damasceno, C. L. Phillips, and S. C. Glotzer, Computational self-assembly of a one-component icosahedral quasicrystal, Nature Materials 14, 109-116 (2015).

[33] T. Dotera, T. Oshiro, and P. Ziherl, Mosaic two-lengthscale quasicrystals, Nature 506, $208-211$ (2014).

[34] A. Yethiraj, Tunable colloids: control of colloidal phase transitions with tunable interactions, Soft Matter 3(9), 1099-1115 (2007).

[35] J. Savage, D. Blair, A. Levine, R. Guyer, and A. Dinsmore, Imaging the sublimation dynamics of colloidal crystallites, Science 314, 795-798 (2006).

[36] L. Feng, B. Laderman, S. Sacanna, and P. Chaikin, Re-entrant solidification in polymer-colloid mixtures as a consequence of competing entropic and enthalpic attractions, Nature Materials 14, 61-65 (2014).

[37] L. Rossi, V. Soni, D. J. Ashton, D. J. Pine, A. P. , P. M. Chaikin, M. Dijkstra, S. Sacanna, and W. T. Irvine, Shapesensitive crystallization in colloidal superball fluids, Proc. Natl. Acad. Sci. U.S.A. 112, 5286-5290 (2015).

[38] J. Savage and A. Dinsmore, Experimental evidence for two-step nucleation in colloidal crystallization, Phys. Rev. Lett. 102, 198302 (2009).

[39] C. Hertlein, L. Helden, A. Gambassi, S. Dietrich, and C. Bechinger, Direct measurement of critical Casimir forces, Nature 451, 172-175 (2008).

[40] S. Faber, Z. Hu, G. H. Wegdam, P. Schall, et al., Controlling colloidal phase transitions with critical Casimir forces, Nature Communications 4, 1584 (2013).

[41] J. R. Edison, N. Tasios, S. Belli, R. Evans, R. van Roij, and M. Dijkstra, Critical casimir forces and colloidal phase transitions in a near-critical solvent: a simple model reveals a rich phase diagram, Phys. Rev. Lett. 114, 038301 (2015).

[42] N. Gnan, E. Zaccarelli, and F. Sciortino, Casimir-like forces at the percolation transition, Nature Communications 5, 3267 (2014).

[43] M. Hagen, E. Meijer, G. Mooij, and D. Frenkel, Does C60 have a liquid phase?, Nature 365, 425-426 (1993).

[44] C. P. Royall, S. R. Williams, T. Ohtsuka, and H. Tanaka, Direct observation of a local structural mechanism for dynamic arrest, Nature Materials 7, 556-561 (2008).

[45] M. Fasolo and P. Sollich, Equilibrium phase behavior of polydisperse hard spheres, Phys. Rev. Lett. 91, 068301 (2003). 
[46] H. Tong, P. Tan, and N. Xu, From Crystals to Disordered Crystals: A Hidden Order-Disorder Transition, Scientific reports 5, 15378 (2015).

[47] D. Kaya, N. Green, C. Maloney, and M. Islam, Normal modes and density of states of disordered colloidal solids, Science 329, 656-658 (2010).

[48] K. Chen, T. Still, S. Schoenholz, K. B. Aptowicz, M. Schindler, A. Maggs, A. J. Liu, and A. Yodh, Phonons in twodimensional soft colloidal crystals, Phys. Rev. E 88, 022315 (2013).

[49] A. Yethiraj, A. Wouterse, B. Groh, and A. van Blaaderen, Nature of an electric-field-induced colloidal martensitic transition, Phys. Rev. Lett. 92, 058301 (2004).

[50] A. F. Demirörs, P. P. Pillai, B. Kowalczyk, and B. A. Grzybowski, Colloidal assembly directed by virtual magnetic moulds, Nature 503, 99-103 (2013).

[51] Y. Roichman and D. Grier, Holographic assembly of quasicrystalline photonic heterostructures, Optics Express 13, 5434-5439 (2005).

[52] H.-D. Deng, G.-C. Li, H.-Y. Liu, Q.-F. Dai, L.-J. Wu, S. Lan, A. V. Gopal, V. A. Trofimov, and T. M. Lysak, Assembling of three-dimensional crystals by optical depletion force induced by a single focused laser beam, Optics Express 20, 9616-9623 (2012).

[53] N. Vogel, L. De Viguerie, U. Jonas, C. K. Weiss, and K. Landfester, Wafer-scale fabrication of ordered binary colloidal monolayers with adjustable stoichiometries, Adv. Func. Mater. 21(16), 3064 ?073 (2011).

[54] T. Neuhaus, M. Marechal, M. Schmiedeberg, and H. Löwen, Rhombic preordering on a square substrate, Phys. Rev. Lett. 110, 118301 (2013).

[55] Y. Peng, Z. Wang, A. M. Alsayed, A. G. Yodh, and Y. Han, Melting of colloidal crystal films, Phys. Rev. Lett. 104, 205703 (2010).

[56] K. Zahn, R. Lenke, and G. Maret, Two-stage melting of paramagnetic colloidal crystals in two dimensions, Phys. Rev. Lett. 82, 2721 (1999).

[57] Q. Wei and X. Wu, Grain boundary dynamics under mechanical annealing in two-dimensional colloids, Phys. Rev. E 70, 020401 (2004).

[58] W. T. Irvine, A. D. Hollingsworth, D. G. Grier, and P. M. Chaikin, Dislocation reactions, grain boundaries, and irreversibility in two-dimensional lattices using topological tweezers, Proc. Natl. Acad. Sci. U.S.A. 110, 15544-15548 (2013).

[59] J. D. Joannopoulos, S. G. Johnson, J. N. Winn, and R. D. Meade, Photonic crystals: molding the flow of light, Princeton University Press, 2011.

[60] E. Matijevic, Monodispersed metal (hydrous) oxides-A fascinating field of colloid science, Acc. Chem. Res. 14, 22-29 (1981).

[61] M. Okubo, M. Ando, A. Yamada, Y. Katsuta, and T. Matsumoto, Studies on suspension and emulsion. XLVII. Anomalous composite polymer emulsion particles with voids produced by seeded emulsion polymerization, J. Polym. Sci. 19, 143-147 (1981).

[62] K. J. Lee, J. Yoon, and J. Lahann, Recent advances with anisotropic particles, Curr. Opin. Colloid Interface Sci. 16, 195-202 (2011).

[63] S. Sacanna and D. J. Pine, Shape-anisotropic colloids: building blocks for complex assemblies, Curr. Opin. Colloid Interface Sci. 16, 96-105 (2011).

[64] M. C. Marchetti, J. F. Joanny, S. Ramaswamy, T. B. Liverpool, J. Prost, M. Rao, and R. A. Simha, Hydrodynamics of soft active matter, Rev. Mod. Phys. 85(3), 1143-1189 (2013).

[65] G.-R. Yi, D. J. Pine, and S. Sacanna, Recent progress on patchy colloids and their self-assembly, J. Phys. Cond. Matter 25, 193101 (2013).

[66] M. Sindoro, N. Yanai, A.-Y. Jee, and S. Granick, Colloidal-sized metal-organic frameworks: synthesis and applications, Acc. Chem. Res. 47, 459-469 (2013).

[67] A. M. Alsayed, M. F. Islam, J. Zhang, P. J. Collings, and A. G. Yodh, Premelting at defects within bulk colloidal crystals, Science 309, 1207-1210 (2005).

[68] C. Zhang, R. J. Macfarlane, K. L. Young, C. H. J. Choi, L. Hao, E. Auyeung, G. Liu, X. Zhou, and C. A. Mirkin, A general approach to DNA-programmable atom equivalents, Nature Materials 12, 741-746 (2013).

[69] P. F. Damasceno, M. Engel, and S. C. Glotzer, Predictive self-assembly of polyhedra into complex structures, Science 337, 453-457 (2012).

[70] A. P. Gantapara, J. de Graaf, R. van Roij, and M. Dijkstra, Phase diagram and structural diversity of a family of truncated cubes: Degenerate close-packed structures and vacancy-rich states, Phys. Rev. Lett. 111, 015501 (2013).

[71] S. A. Rice, A. R. Dinner, and M. Dijkstra, Entropy-driven phase transitions in colloids: from spheres to anisotropic particles, Adv. Chem. Phys. 156, 35 (2015).

[72] S. C. Glotzer and M. J. Solomon, Anisotropy of building blocks and their assembly into complex structures, Nature Materials 6, 557-562 (2007).

[73] F. Li, D. P. Josephson, and A. Stein, Colloidal assembly: the road from particles to colloidal molecules and crystals, Angew. Chem. Inter. Edit. 50, 360-388 (2011).

[74] J. Zhang, E. Luijten, and S. Granick, Toward design rules of directional janus colloidal assembly, Annual review of physical chemistry 66, 581-600 (2015).

[75] G. Bautista-Carbajal and G. Odriozola, Phase diagram of two-dimensional hard ellipses, J. Chem. Phys. 140, 204502 (2014).

[76] G. Bautista-Carbajal, A. Moncho-Jordá, and G. Odriozola, Further details on the phase diagram of hard ellipsoids of 
revolution, J. Chem. Phys. 138, 064501 (2013).

[77] Z. Zheng, F. Wang, and Y. Han, Glass transitions in quasi-two-dimensional suspensions of colloidal ellipsoids., Phys. Rev. Lett. 107, 065702 (2011).

[78] B. Liu, T. H. Besseling, M. Hermes, A. F. Demirörs, A. Imhof, and A. van Blaaderen, Switching plastic crystals of colloidal rods with electric fields, Nature Communications 5, 3092 (2014).

[79] C. J. Hernandez and T. G. Mason, Colloidal alphabet soup: monodisperse dispersions of shape-designed lithoparticles, J. Phys. Chem. C 111, 4477-4480 (2007).

[80] K. Zhao, R. Bruinsma, and T. G. Mason, Entropic crystal-crystal transitions of Brownian squares, Proc. Natl. Acad. Sci. U.S.A. 108, 2684-2687 (2011).

[81] K. Zhao and T. G. Mason, Self-organized chiral colloidal crystals of Brownian square crosses, J. Phys. Cond. Matter 26, 152101 (2014).

[82] K. Zhao, R. Bruinsma, and T. G. Mason, Local chiral symmetry breaking in triatic liquid crystals, Nature Communications , 801 (2012).

[83] A. P. Gantapara, W. Qi, and M. Dijkstra, A novel chiral phase of achiral hard triangles and an entropy-driven demixing of enantiomers, Soft Matter 11, 8684-8691 (2015).

[84] L. Rossi, S. Sacanna, W. T. Irvine, P. M. Chaikin, D. J. Pine, and A. P. Philipse, Cubic crystals from cubic colloids, Soft Matter 7, 4139-4142 (2011).

[85] H. R. Vutukuri, A. Imhof, and A. Van Blaaderen, Fabrication of Polyhedral Particles from Spherical Colloids and Their Self-Assembly into Rotator Phases, Angew. Chem. Int. Ed. 53, 13830-13834 (2014).

[86] J. Henzie, M. Grünwald, A. Widmer-Cooper, P. L. Geissler, and P. Yang, Self-assembly of uniform polyhedral silver nanocrystals into densest packings and exotic superlattices, Nature Materials 11, 131-137 (2012).

[87] K. Miszta, J. de Graaf, G. Bertoni, D. Dorfs, R. Brescia, S. Marras, L. Ceseracciu, R. Cingolani, R. van Roij, M. Dijkstra, et al., Hierarchical self-assembly of suspended branched colloidal nanocrystals into superlattice structures, Nature Materials 10, 872-876 (2011).

[88] S. Jiang, Q. Chen, M. Tripathy, E. Luijten, K. S. Schweizer, and S. Granick, Janus particle synthesis and assembly, Adv. Mater. 22, 1060-1071 (2010).

[89] A. Walther and A. H. Muller, Janus particles: synthesis, self-assembly, physical properties, and applications, Chem. Rev. 113, 5194-5261 (2013).

[90] Y. Wang, Y. Wang, D. R. Breed, V. N. Manoharan, L. Feng, A. D. Hollingsworth, M. Weck, and D. J. Pine, Colloids with valence and specific directional bonding, Nature 491, 51-55 (2012).

[91] Q. Chen, S. C. Bae, and S. Granick, Directed self-assembly of a colloidal kagome lattice, Nature 469, 381-384 (2011).

[92] X. Mao, Q. Chen, and S. Granick, Entropy favours open colloidal lattices, Nature Materials 12, $217-222$ (2013).

[93] A. W. Wilber, J. P. Doye, A. A. Louis, E. G. Noya, M. A. Miller, and P. Wong, Reversible self-assembly of patchy particles into monodisperse icosahedral clusters, J. Chem. Phys. 127, 085106 (2007).

[94] F. Romano, E. Sanz, P. Tartaglia, and F. Sciortino, Phase diagram of trivalent and pentavalent patchy particles, J. Phys. Cond. Matter 24, 064113 (2012).

[95] F. Romano and F. Sciortino, Patterning symmetry in the rational design of colloidal crystals, Nature Communications 3, 975 (2012).

[96] H. Shin and K. S. Schweizer, Theory of two-dimensional self-assembly of Janus colloids: crystallization and orientational ordering, Soft Matter 10, 262-274 (2014).

[97] S. Whitelam and R. L. Jack, The statistical mechanics of dynamic pathways to self-assembly, Annu. Rev. Phys. Chem. 66, 143-163 (2015).

[98] T. Vissers, Z. Preisler, F. Smallenburg, M. Dijkstra, and F. Sciortino, Predicting crystals of Janus colloids, J. Chem. Phys. 138, 164505 (2013).

[99] W. B. Rogers and V. N. Manoharan, Programming colloidal phase transitions with DNA strand displacement, Science 347, 639-642 (2015).

[100] M. T. Casey, R. T. Scarlett, W. B. Rogers, I. Jenkins, T. Sinno, and J. C. Crocker, Driving diffusionless transformations in colloidal crystals using DNA handshaking, Nature Communications 3, 1209 (2012).

[101] E. Auyeung, T. I. Li, A. J. Senesi, A. L. Schmucker, B. C. Pals, M. O. de La Cruz, and C. A. Mirkin, DNA-mediated nanoparticle crystallization into Wulff polyhedra, Nature 505, 73-77 (2014).

[102] W. M. Jacobs, A. Reinhardt, and D. Frenkel, Rational design of self-assembly pathways for complex multicomponent structures, Proc. Natl. Acad. Sci. U.S.A. 112, 6313-6318 (2015).

[103] A. Reinhardt and D. Frenkel, Numerical evidence for nucleated self-assembly of DNA brick structures, Phys. Rev. Lett. 112, 238103 (2014).

[104] J. de Graaf, R. van Roij, and M. Dijkstra, Dense regular packings of irregular nonconvex particles, Phys. Rev. Lett. 107, 155501 (2011).

[105] B. Senyuk, Q. Liu, S. He, R. D. Kamien, R. B. Kusner, T. C. Lubensky, and I. I. Smalyukh, Topological colloids, Nature 493, 200-205 (2013).

[106] U. Tkalec, M. Ravnik, S. Čopar, S. Žumer, and I. Muševič, Reconfigurable knots and links in chiral nematic colloids, Science 333, 62-65 (2011).

[107] Y. Hong, D. Velegol, N. Chaturvedi, and A. Sen, Biomimetic behavior of synthetic particles: from microscopic randomness to macroscopic control, Phys. Chem. Chem. Phys. 12, 1423-1435 (2010).

[108] I. Buttinoni, J. Bialké, F. Kümmel, H. Löwen, C. Bechinger, and T. Speck, Dynamical clustering and phase separation in suspensions of self-propelled colloidal particles, Phys. Rev. Lett. 110, 238301 (2013). 
[109] A. P. Petroff, X.-L. Wu, and A. Libchaber, Fast-moving bacteria self-organize into active two-dimensional crystals of rotating cells, Phys. Rev. Lett. 114, 158102 (2015).

[110] J. Yan, S. C. Bae, and S. Granick, Rotating crystals of magnetic Janus colloids, Soft Matter 11, 147-153 (2015).

[111] J. Palacci, S. Sacanna, A. P. Steinberg, D. J. Pine, and P. M. Chaikin, Living crystals of light-activated colloidal surfers, Science 339, 936-940 (2013).

[112] F. Ginot, I. Theurkauff, D. Levis, C. Ybert, L. Bocquet, L. Berthier, and C. Cottin-Bizonne, Nonequilibrium equation of state in suspensions of active colloids, Phys. Rev. X 5, 011004 (2015).

[113] D. J. Aastuen, N. A. Clark, L. K. Cotter, and B. J. Ackerson, Nucleation and growth of colloidal crystals., Phys. Rev. Lett. 57(14), 1733-1736 (1986).

[114] R. P. Sear, Nucleation: theory and applications to protein solutions and colloidal suspensions, J. Phys. Cond. Matt. 19, 033101 (2007).

[115] A. Samanta, M. E. Tuckerman, T.-Q. Yu, and E. Weinan, Microscopic mechanisms of equilibrium melting of a solid, Science 346, 729-732 (2014).

[116] W. Qi, Y. Peng, Y. Han, R. K. Bowles, and M. Dijkstra, Nonclassical Nucleation in a Solid-Solid Transition of Confined Hard Spheres, Phys. Rev. Lett. 115, 185701 (2015).

[117] G. Meng, J. Paulose, D. R. Nelson, and V. N. Manoharan, Elastic instability of a crystal growing on a curved surface, Science 343, 634-637 (2014).

[118] P. Tan, N. Xu, and L. Xu, Visualizing kinetic pathways of homogeneous nucleation in colloidal crystallization, Nature Physics 10, 73-79 (2014).

[119] N. M. Dixit and C. F. Zukoski, Kinetics of crystallization in hard-sphere colloidal suspensions, Phys. Rev. E 64, 041604 (2001).

[120] J. F. Lutsko and M. A. Durán-Olivencia, Classical nucleation theory from a dynamical approach to nucleation, J. Chem. Phys. 138, 244908 (2013).

[121] Y. Peng, F. Wang, Z. Wang, A. M. Alsayed, Z. Zhang, A. G. Yodh, and Y. Han, Two-step nucleation mechanism in solid-solid phase transitions, Nature Materials 14, 101-108 (2015).

[122] Y. Yang, L. Fu, C. Marcoux, J. E. Socolar, P. Charbonneau, and B. B. Yellen, Phase transformations in binary colloidal monolayers, Soft Matter 11, 2404-2415 (2015).

[123] W. B. Rogers and J. C. Crocker, Direct measurements of DNA-mediated colloidal interactions and their quantitative modeling, Proc. Natl. Acad. Sci. U.S.A. 108, 15687-15692 (2011).

[124] Z. Zeravcic, V. N. Manoharan, and M. P. Brenner, Size limits of self-assembled colloidal structures made using specific interactions, Proc. Natl. Acad. Sci. U.S.A. 111, 15918-15923 (2014).

[125] J. J. Crassous, A. M. Mihut, L. K. Månsson, and P. Schurtenberger, Anisotropic responsive microgels with tuneable shape and interactions, Nanoscale 7, 15971-15982 (2015).

[126] E. Canovi, P. Werner, and M. Eckstein, First-order dynamical phase transitions, Phys. Rev. Lett. 113, 265702 (2014).

[127] A. Del Campo and W. H. Zurek, Universality of phase transition dynamics: Topological defects from symmetry breaking, Inter. J. Mod. Phys. A 29, 1430018 (2014).

[128] U. Gasser, Crystallization in three-and two-dimensional colloidal suspensions, J. Phys. Cond. Matter 21, 203101 (2009).

[129] T. Palberg, Crystallization kinetics of colloidal model suspensions: recent achievements and new perspectives, J. Phys. Cond. Matter 26, 333101 (2014).

[130] U. Gasser, E. R. Weeks, A. Schofield, P. Pusey, and D. Weitz, Real-space imaging of nucleation and growth in colloidal crystallization, Science 292, 258-262 (2001).

[131] S. Auer and D. Frenkel, Prediction of absolute crystal-nucleation rate in hard-sphere colloids, Nature 409, 1020-1023 (2001).

[132] T. Kawasaki and H. Tanaka, Formation of a crystal nucleus from liquid, Proc. Natl. Acad. Sci. U.S.A. 107, 14036-14041 (2010).

[133] S. Alexander and J. McTague, Should all crystals be bcc? Landau theory of solidification and crystal nucleation, Phys. Rev. Lett. 41, 702 (1978).

[134] T. Schilling, H. Schöpe, M. Oettel, G. Opletal, and I. Snook, Precursor-mediated crystallization process in suspensions of hard spheres, Phys. Rev. Lett. 105, 025701 (2010).

[135] Y. Wang, Y. Wang, X. Zheng, É. Ducrot, J. S. Yodh, M. Weck, and D. J. Pine, Crystallization of DNA-coated colloids, Nature Communications 6, 7253 (2015).

[136] S. Auer and D. Frenkel, Line tension controls wall-induced crystal nucleation in hard-sphere colloids, Phys. Rev. Lett. 91, 015703 (2003).

[137] M. Dijkstra, Capillary freezing or complete wetting of hard spheres in a planar hard slit?, Phys. Rev. Lett. 93, 108303 (2004).

[138] G. I. Tóth, G. Tegze, T. Pusztai, and L. Gránásy, Heterogeneous crystal nucleation: the effect of lattice mismatch, Phys. Rev. Lett. 108, 025502 (2012).

[139] A. Cacciuto, S. Auer, and D. Frenkel, Onset of heterogeneous crystal nucleation in colloidal suspensions, Nature 428, 404-406 (2004).

[140] E. Allahyarov, K. Sandomirski, S. Egelhaaf, and H. Löwen, Crystallization seeds favour crystallization only during initial growth, Nature Communications 6, 7110 (2015).

[141] M. Hermes, E. Vermolen, M. Leunissen, D. Vossen, P. van Oostrum, M. Dijkstra, and A. van Blaaderen, Nucleation of colloidal crystals on configurable seed structures, Soft Matter 7, 4623-4628 (2011). 
[142] B. Halperin and D. R. Nelson, Theory of two-dimensional melting, Phys. Rev. Lett. 41, 121 (1978).

[143] A. Young, Melting and the vector Coulomb gas in two dimensions, Phys. Rev. B 19, 1855 (1979).

[144] S. Chui, Grain-boundary theory of melting in two dimensions, Phys. Rev. Lett. 48, 933 (1982).

[145] S. Deutschländer, P. Dillmann, G. Maret, and P. Keim, Kibble-Zurek mechanism in colloidal monolayers, Proc. Natl. Acad. Sci. U.S.A. 112, 6925-6930 (2015).

[146] W. T. Irvine, V. Vitelli, and P. M. Chaikin, Pleats in crystals on curved surfaces, Nature 468, 947-951 (2010).

[147] E. Zaccarelli, C. Valeriani, E. Sanz, W. Poon, M. Cates, and P. Pusey, Crystallization of hard-sphere glasses, Phys. Rev. Lett. 103, 135704 (2009).

[148] E. Sanz, C. Valeriani, E. Zaccarelli, W. C. Poon, P. N. Pusey, and M. E. Cates, Crystallization mechanism of hard sphere glasses, Phys. Rev. Lett. 106, 215701 (2011).

[149] X.-M. Bai and M. Li, Nature and extent of melting in superheated solids: Liquid-solid coexistence model, Phys. Rev. B 72, 052108 (2005).

[150] Y. Peng, Z. Wang, and Y. Han, Melting of microgel colloidal crystals, J. Phys. Conf. Seri. 319, 012010 (2011).

[151] J. Dash, A. Rempel, and J. Wettlaufer, The physics of premelted ice and its geophysical consequences, Rev. Mod. Phys. 78, 695 (2006).

[152] B. LI, F. Wang, Y. Peng, R. Ni, and Y. Han, Modes of surface premelting in colloidal crystals composed of attractive particles, Nature, doi:10.1038/nature16987 (2016).

[153] B. Van Der Meer, W. Qi, R. G. Fokkink, J. Van Der Gucht, M. Dijkstra, and J. Sprakel, Highly cooperative stress relaxation in two-dimensional soft colloidal crystals, Proc. Natl. Acad. Sci. U.S.A. 111, 15356-15361 (2014).

[154] A. Cavagna, Supercooled ciquids for pedestrians, Phys. Rep. 476, 51 ?24 (2009).

[155] Y. Han, N. Ha, A. Alsayed, and A. Yodh, Melting of two-dimensional tunable-diameter colloidal crystals, Phys. Rev. E 77, 041406 (2008).

[156] C. Mak, Large-scale simulations of the two-dimensional melting of hard disks, Phys. Rev. E 73, 065104 (2006).

[157] S. C. Kapfer and W. Krauth, Two-dimensional melting: from liquid-hexatic coexistence to continuous transitions, Phys. Rev. Lett. 114, 035702 (2015).

[158] E. P. Bernard and W. Krauth, Two-step melting in two dimensions: first-order liquid-hexatic transition, Phys. Rev. Lett. 107, 155704 (2011).

[159] D.-M. Zhu, D. Pengra, and J. Dash, Edge melting in two-dimensional solid films, Phys. Rev. B 37, 5586 (1988).

[160] Y. Peng, Z.-R. Wang, A. M. Alsayed, A. G. Yodh, Y. Han, et al., Melting of multilayer colloidal crystals confined between two walls, Phys. Rev. E 83, 011404 (2011).

[161] P. Tolédano and V. Dmitriev, Reconstructive phase transitions: in crystals and quasicrystals, World Scientific, 1996.

[162] J. A. Weiss, D. W. Oxtoby, D. G. Grier, and C. A. Murray, Martensitic transition in a confined colloidal suspension, J. Chem. Phys. 103, 1180-1190 (1995).

[163] P. Bolhuis and D. Frenkel, Prediction of an expanded-to-condensed transition in colloidal crystals, Phys. Rev. Lett. 72, 2211 (1994).

[164] J. Bai, C. A. Angell, and X. C. Zeng, Guest-free monolayer clathrate and its coexistence with two-dimensional high-density ice, Proc. Natl. Acad. Sci. U.S.A. 107, 5718-5722 (2010).

[165] P. S. Mohanty, P. Bagheri, S. Nöjd, A. Yethiraj, and P. Schurtenberger, Multiple path-dependent routes for phasetransition kinetics in thermoresponsive and field-pesponsive ultrasoft Colloids, Phys. Rev. X 5, 011030 (2015).

[166] A. E. Larsen and D. G. Grier, Melting of metastable crystallites in charge-stabilized colloidal suspensions, Phys. Rev. Lett. 76, 3862 (1996).

[167] V. Thapar and F. A. Escobedo, Localized orientational order chaperones the nucleation of rotator phases in hard polyhedral particles, Phys. Rev. Lett. 112, 048301 (2014).

[168] J. Bialké, T. Speck, and H. Löwen, Crystallization in a dense suspension of self-propelled particles, Phys. Rev. Lett. 108, 168301 (2012).

[169] A. M. Menzel and H. Löwen, Traveling and resting crystals in active systems, Phys. Rev. Lett. 110, 055702 (2013).

[170] C. A. Weber, C. Bock, and E. Frey, Defect-mediated phase transitions in active soft matter, Phys. Rev. Lett. 112, 168301 (2014).

[171] Y. Goto and H. Tanaka, Purely hydrodynamic ordering of rotating disks at a finite Reynolds number, Nature Communications 6, 5994 (2015).

[172] A. Zöttl and H. Stark, Hydrodynamics determines collective motion and phase behavior of active colloids in quasi-twodimensional confinement, Phys. Rev. Lett. 112, 118101 (2014).

[173] H. H. Malte Henkel, Non-equilibrium phase transitions, Springer, 2014.

[174] Y. L. Wu, D. Derks, A. van Blaaderen, and A. Imhof, Melting and crystallization of colloidal hard-sphere suspensions under shear, Proc. Natl. Acad. Sci. U.S.A. 106, 10564-10569 (2009).

[175] X. Cheng, X. Xu, S. A. Rice, A. R. Dinner, and I. Cohen, Assembly of vorticity-aligned hard-sphere colloidal strings in a simple shear flow, Proc. Natl. Acad. Sci. U.S.A. 109, 63-67 (2012).

[176] K.-Q. Zhang and X. Y. Liu, In situ observation of colloidal monolayer nucleation driven by an alternating electric field, Nature 429, 739-743 (2004).

[177] Z. Hu, P. Schall, et al., Single crystal growth and anisotropic crystal-fluid interfacial free energy in soft colloidal systems, Phys. Rev. E 84, 011607 (2011).

[178] M. R. Jones, N. C. Seeman, and C. A. Mirkin, Programmable materials and the nature of the DNA bond, Science 347, 840-842 (2015).

[179] J. R. Tumbleston, D. Shirvanyants, N. Ermoshkin, R. Janusziewicz, A. R. Johnson, D. Kelly, K. Chen, R. Pinschmidt, 
J. P. Rolland, A. Ermoshkin, et al., Continuous liquid interface production of 3D objects, Science 347, 1349-1352 (2015).

[180] A. Pérez-Escudero, J. Vicente-Page, R. C. Hinz, S. Arganda, and G. G. de Polavieja, idTracker: tracking individuals in a group by automatic identification of unmarked animals, Nature Methods 11(7), 743-748 (2014).

[181] W. E. Moerner, Single-molecule spectroscopy, imaging, and photocontrol: foundations for super-resolution microscopy (nobel lecture), Ang. Chem. Inter. Edit. 54, 8067-8093 (2015).

[182] J. Park, H. Elmlund, P. Ercius, J. M. Yuk, D. T. Limmer, Q. Chen, K. Kim, S. H. Han, D. A. Weitz, A. Zettl, et al., 3D structure of individual nanocrystals in solution by electron microscopy, Science 349, 290-295 (2015).

[183] Editorial, Method of the Year 2014, Nature Methods 12, 1 (2015).

[184] B.-C. Chen, W. R. Legant, K. Wang, L. Shao, D. E. Milkie, M. W. Davidson, C. Janetopoulos, X. S. Wu, J. A. Hammer, Z. Liu, et al., Lattice light-sheet microscopy: Imaging molecules to embryos at high spatiotemporal resolution, Science 346, 125-132 (2014). 